\title{
Allergic bronchopulmonary aspergillosis screening in bronchiectasis: is there always a precise answer to a clear question?
}

Cardio-Thoracic-Vascular Department, University of Milano Bicocca, Respiratory Unit, San Gerardo Hospital, ASST Monza, Monza, Italy

\section{KEY WORDS}

bronchiectasis; allergic bronchopulmonary aspergillosis;

severe asthma with fungal sensitisation; Aspergillus-

associated syndromes; bronchiectasis screening

\section{Corresponding Author}

Paola Faverio

Cardio-Thoracic-Vascular Department

University of Milano Bicocca

Respiratory Unit, San Gerardo Hospital

ASST Monza, Via Pergolesi 33

20900, Monza, Italy

Phone: +393382185092

Fax: +390392336660

E-mail: paola.faverio@gmail.com

Doi

10.23822/EurAnnACI.1764-1489.62

\section{To the Editor}

The most recently published guidelines about the management of adult bronchiectasis suggest screening all patients for allergic bronchopulmonary aspergillosis (ABPA), since this is a potentially treatable disease and modifiable cause of bronchiectasis (1). Tests recommended include total serum IgE, specific IgG to Aspergillus and specific IgE to Aspergillus or, as an alternative, skin prick tests to Aspergillus.

ABPA prevalence in adults with bronchiectasis is low, and varies between 1 and $11 \%$ in different cohorts $(2,3)$. However, in some cases, tests for ABPA might be altered but not consistent with a diagnosis of ABPA. No recommendations are available about management of these patients with only some nonspe- cific serological alterations suggestive of ABPA, and about their risk to develop ABPA or other Aspergillus-associated syndromes, such as severe asthma with fungal sensitisation (SAFS) (4), during follow-up.

To answer these questions, we retrospectively reviewed our prospectively-collected cohort of consecutive adult patients with non-cystic fibrosis bronchiectasis attending the outpatient clinic at the San Gerardo Hospital, Monza, Italy, from January $1^{\text {st }}$, 2013 to December 1 ${ }^{\text {st }}, 2016$ (Institutional Review Board approval n. 234, September 2013). To define ABPA we used the diagnostic criteria proposed by the ABPA Working Group ISHAM 2013 (5). After screening visit, all patients were offered a clinical and functional follow-up at our outpatient clinic. Clinical deterioration was established by the attending physicians, 
Figure 1 - Flow chart of study population.

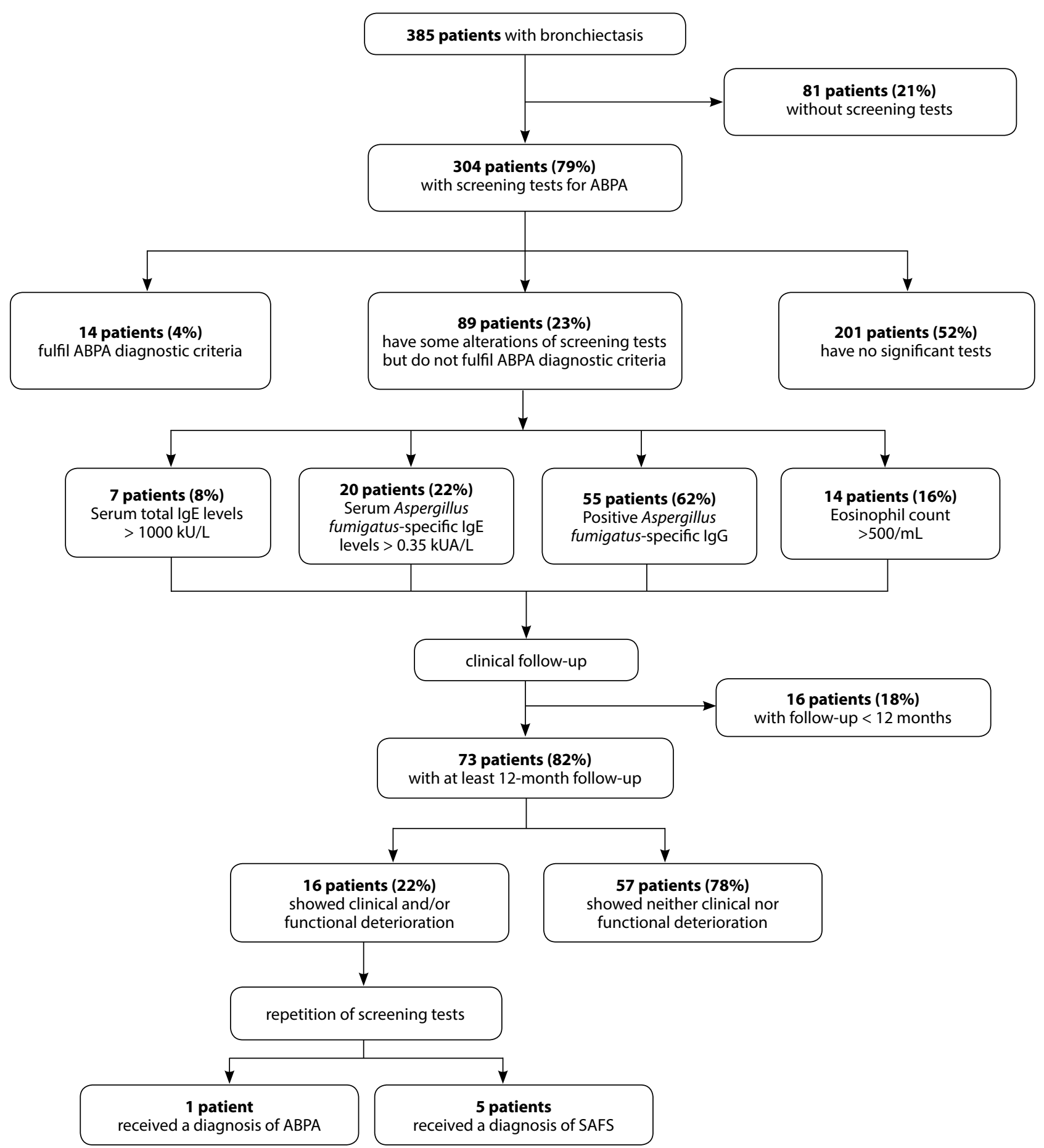


according to the following parameters: onset or worsening of respiratory symptoms, such as cough, dyspnea and sputum production, or pulmonary exacerbations $\geq 2 /$ year. Functional deterioration was defined as Forced Expiratory Volume in the 1st second (FEV1) and/or Forced Vital Capacity (FVC) > 10\% reduction compared to the prior test.

Out of 385 patients in our cohort, the prevalence of ABPA was $4 \%$, consistent with the prior literature (2-3) (figure 1). Almost one quarter $(89,23 \%)$ of our patients presented, at the time of first evaluation, one or more alterations of ABPA testing (figure 1). Eighteen (20\%) patients showed multiple contemporary alterations of screening exams.

Out of 73 patients with nonspecific ABPA screening alterations at baseline and at least a 12-month clinical follow-up (median follow-up time 25 months), the majority (57 patients, 78\%) showed neither clinical nor functional deterioration and none of them developed Aspergillus-associated syndromes, while a minority of patients $(16-22 \%)$ repeated screening tests because of clinical and/or functional deterioration. In these patients the repetition of screening tests, together with functional and clinical evolution, allowed to make a diagnosis of ABPA in 1 case and SAFS in 5 cases.

In conclusion, only a minority of bronchiectasis patients (4\%) were diagnosed with ABPA at baseline, while nonspecific alterations of screening tests not consistent with a diagnosis of ABPA were common (almost one quarter of cases in our cohort). In this specific group, $22 \%$ of patients showed clinical and/or function- al deterioration during follow-up and almost $8 \%$ received a diagnosis of ABPA or SAFS. Therefore, a non-negligible proportion of patients with nonspecific alterations of screening tests at baseline may develop Aspergillus-associated syndromes during follow-up. Such condition should be suspected in particular in the presence of clinical and/or functional deterioration. Further studies on multi-centric cohorts and longer follow-up periods are needed to confirm our observations.

\section{Conflict of interest}

The authors declare that they have no conflict of interest.

\section{References}

1. Polverino E, Goeminne PC, McDonnell MJ, Aliberti S, Marshall SE, Loebinger M, et al. European Respiratory Society guidelines for the management of adult bronchiectasis. Eur Respir J 2017; 9:50(3).

2. De Soyza A, Aliberti S. Bronchiectasis and Aspergillus: How are they linked? Med Mycol 2017; 55(1):69-81.

3. Suarez-Cuartin G, Chalmers JD, Sibila O. Diagnostic challenges of bronchiectasis. Respir Med 2016; 116:70-77.

4. Agarwal R. Severe asthma with fungal sensitization. Curr Allergy Asthma Rep 2011; 11:403-413.

5. Agarwal R, Chakrabarti A, Shah A, Gupta D, Meis JF, Guleria R, et al. Allergic bronchopulmonary aspergillosis: review of literature and proposal of new diagnostic and classification criteria. Clin Exp Allergy 2013; 43(8):850-873. 